Pacific Journal of Mathematics

RADIAL DISTRIBUTION AND DEFICIENCIES OF THE 


\title{
RADIAL DISTRIBUTION AND DEFICIENCIES OF THE VALUES OF A MEROMORPHIC FUNCTION
}

\author{
Albert Edrei ${ }^{1}$, Wolfgang H. J. Fuchs ${ }^{2}$ \\ AND Simon Hellerstein
}

Introduction. Let $f(z)$ be a meromorphic function. Throughout this note we make the following conventions.

I. $f(0)=1$; this simplifies the exposition without affecting the generality of the results.

II. We denote by

$$
a_{1}, a_{2}, a_{3}, \cdots
$$

the sequence of the zeros of $f(z)$ and by

$$
b_{1}, b_{2}, b_{3}, \cdots
$$

the sequence of its poles.

The moduli of the terms of these two sequences are taken to be nondecreasing and each zero or pole appears as often as indicated by its multiplicity.

III. The standard symbols of Nevanlinna's theory:

$$
\log ^{+}, m(r, f), \log M(r, f), n(r, f), N(r, f), T(r, f), \delta(\tau, f)
$$

are used systematically; familiarity with their meaning is assumed.

We investigate here the following problem, a special case of which has already been mentioned by two of the authors $[1 ; \mathrm{p} .295]$ :

To find sequences $\left\{a_{\mu}\right\},\left\{b_{\nu}\right\}$ such that if $f(z)$ is a meromorphic function with zeros $\left\{a_{\mu}\right\}$ and poles $\left\{b_{\nu}\right\}$ (and no other zeros or poles), then

$$
\delta(0, f)>0, \quad \delta(\infty, f)>0 .
$$

The results of the present note show that a simple behavior of the arguments of the zeros and poles is almost sufficient to induce the inequalities (1). We prove

THEOREM 1. Let $f(z)$ be a meromorphic function with positive zeros and negative poles.

Received April 25, 1960.

1 The research of this author was supported by the United States Air Force, Contract No. AF49(638)-571, monitored by the Office of Scientific Research.

2 The research of this author was supported by a grant from the National Science Foundation (G 5253). 
Assume that

$$
\sum_{\mu} \frac{1}{a_{\mu}}+\sum_{\nu} \frac{1}{\left|b_{\nu}\right|}=+\infty
$$

and that

$$
\sum_{\mu} \frac{1}{a_{\mu}^{\xi}}+\sum_{\nu} \frac{1}{\left|b_{\nu}\right|^{\xi}}<+\infty
$$

for some finite positive value of $\xi$.

Then

$$
\limsup _{r \rightarrow \infty} \frac{N\left(r, \frac{1}{f}\right)+N(r, f)}{T(r, f)} \leqq \frac{1}{1+A},
$$

where $A(>0)$ is an absolute constant.

If the condition (3) is omitted, we still have

$$
\limsup _{r \rightarrow \infty} \frac{N\left(r, \frac{1}{f}\right)+N(r, f)}{T(r, f)} \leqq 1 .
$$

COROLlary 1.1. The assumptions of Theorem $1 \mathrm{imply}$

$$
\delta(0, f) \geqq \frac{A}{1+A}, \quad \delta(\infty, f) \geqq \frac{A}{1+A} .
$$

If the condition (3) is omitted, but

$$
0<\alpha \leqq \liminf _{r \rightarrow \infty} \frac{N(r, f)}{N\left(r, \frac{1}{f}\right)} \leqq \limsup _{r \rightarrow \infty} \frac{N(r, f)}{N\left(r, \frac{1}{f}\right)} \leqq \frac{1}{\beta}<+\infty,
$$

we still have

$$
\delta(0, f) \geqq \frac{\alpha}{1+\alpha}, \quad \delta(\infty, f) \geqq \frac{\beta}{1+\beta} .
$$

COROLLARY 1.2. Let $f(z)$ be an entire function with real zeros. If

$$
\sum_{\mu} \frac{1}{\left|a_{\mu}\right|^{2}}=+\infty
$$

and if

$$
\sum_{\mu} \frac{1}{\left|a_{\mu}\right|^{\xi}}<+\infty,
$$


for some finite positive value of $\xi$, then

$$
\delta(0, f) \geqq \frac{A}{1+A},
$$

where $A$ is the absolute constant in (4).

The condition (2) of Theorem 1 cannot be omitted; we shall see that the theorem does not hold for certain meromorphic functions of finite order, with positive poles and such that

$$
\sum_{\mu} \frac{1}{a_{\mu}^{\kappa}}+\sum_{\nu} \frac{1}{\left|b_{\nu}\right|^{\kappa}}=+\infty
$$

for every $\kappa$ less than one.

Similarly, Corollary 1.2 does not hold for certain entire functions of finite order, with real zeros and such that

$$
\sum_{\mu} \frac{1}{\left|a_{\mu}\right|^{\kappa}}=+\infty
$$

for every $\kappa$ less than two.

The conditions (3) and (7) are used essentially in our proofs, but it is possible that our results hold without such restrictions. This conjecture is plausible if we observe that the assertions (4) and (8) do not contain the parameter $\xi$.

Our method gives a little more than has been stated. In the special case of entire functions it yields

THEOREM 2. Let $f(z)$ be entire. Assume that all its zeros $a_{\mu}$ lie on the radii defined by

$$
r e^{i \omega_{0}}, r e^{i \omega_{1}}, \cdots r e^{i \omega_{m}} \quad(r>0),
$$

where the $\omega$ 's are real.

Then, there exists a positive constant $K$, depending only on the $\omega$ 's, and such that the condition

$$
\sum_{\mu} \frac{1}{\left|a_{\mu}\right|^{K}}=+\infty
$$

and the condition

$$
\sum_{\mu} \frac{1}{\left|a_{\mu}\right|^{\xi}}<+\infty
$$

for some finite value of $\xi$, imply

$$
\delta(0, f) \geqq \frac{A}{1+A},
$$


where $A$ is the absolute constant in (4).

All the previous theorems and corollaries assert that 0 and $\infty$ are among the deficient values of certain functions $f(z)$.

Hence, by Theorem 4 of [1], the lower order $\mu$, of $f(z)$ is positive ${ }^{3}$.

Assume now that $h(z)$ denotes a meromorphic function which does not vanish identically, is of order less than $\mu$, but is otherwise arbitrary. Then, by elementary inequalities of Nevanlinna's theory,

$$
\begin{aligned}
& T(r, h f) \sim T(r, f), \\
& \frac{m(r, f h)}{T(r, f h)}=\frac{m(r, f)}{T(r, f)}+o(1), \frac{m\left(r, \frac{1}{f h}\right)}{T(r, f h)}=\frac{m\left(r, \frac{1}{f}\right)}{T(r, f)}+o(1),
\end{aligned}
$$

and hence

$$
\delta(0, f h)=\delta(0, f), \quad \delta(\infty, f h)=\delta(\infty, f) .
$$

This shows that our theorems remain true even if infinitely many zeros and poles have unknown arguments but are sufficiently rare.

It will be shown in [2] that a radial distribution of zeros and poles makes it, in general, impossible for the function to have other deficient values than 0 and $\infty$. Combining the results of [2] with those of the present investigation, it is possible to obtain information concerning all the deficient values of certain interesting classes of functions. The following result is one of the simplest which may be obtained in this way.

Let $f(z)$ be an entire function of finite order $\lambda$. Assume that all the zeros of $f(z)$ are real and that $\lambda .>2$.

Then (11) holds and

$$
\delta(\tau, f)=0
$$

for $\tau \neq 0, \tau \neq \infty$.

\section{Consequences of an identity of Nevanlinna.}

LEMma 1. Let $f(z)$ be meromorphic with zeros $\left\{a_{\mu}\right\}$ and poles $\left\{b_{\nu}\right\}$. Assume

$$
\begin{aligned}
\left|\arg a_{\mu}\right| \leqq \gamma<\frac{\pi}{2} & (\mu=1,2,3, \cdots) ; \\
\left|\arg b_{\nu}-\pi\right| \leqq \gamma<\frac{\pi}{2} & (\nu=1,2,3, \cdots) ;
\end{aligned}
$$

${ }^{3}$ A direct study of the lower order of our functions will be found in [2]. For the functions in Theorem 1 and its Corollaries, this study yields "best possible" bounds for $\mu$. 


$$
\sum_{\mu} \frac{1}{\left|a_{\mu}\right|}+\sum_{\nu} \frac{1}{\left|b_{\nu}\right|}=+\infty
$$

Then, for $r$ large enough,

$$
\frac{1}{2 \pi} \int_{0}^{2 \pi} \log \left|f\left(r e^{i \theta}\right)\right| \cos \theta d \theta \geqq \cos \gamma\left\{N\left(r, \frac{1}{f}\right)+N(r, f)\right\} .
$$

Proof. Put $q=0, z=0$ in a well-known identity of $R$. Nevanlinna [3; p. 222]. Adapting the formula to our notation, we obtain

$$
\left.\begin{array}{c}
\frac{1}{2 \pi} \int_{0}^{2 \pi} \log \left|f\left(r e^{i \theta}\right)\right| e^{-i \theta} d \theta=\frac{r}{2}\left\{\sum _ { | \alpha _ { \mu } | \leqq r } \left(\frac{1}{a_{\mu}}-\overline{\overline{a_{\mu}}}\right.\right. \\
r^{2}
\end{array}\right)
$$

and hence, in view of the assumptions (1.1) and (1.2)

$$
\begin{gathered}
\frac{1}{2 \pi} \int_{0}^{2 \pi} \log \left|f\left(r e^{i \theta}\right)\right| \cos \theta d \theta \geqq \frac{r}{2} \cos \gamma\left\{\sum_{\left|a_{\mu}\right| \leqq r}\left(\frac{1}{\left|a_{\mu}\right|}-\frac{\left|a_{\mu}\right|}{r^{2}}\right)\right. \\
\left.+\sum_{\left|b_{\nu}\right| \leqq r}\left(\frac{1}{\left|b_{\nu}\right|}-\frac{\left|b_{\nu}\right|}{r^{2}}\right)\right\}-\left|\frac{f^{\prime}(0)}{f(0)}\right| \frac{r}{2} .
\end{gathered}
$$

An elementary evaluation yields

$$
\frac{r}{2} \sum_{\mid a_{\mu} \backslash \leq r}\left(\frac{1}{\left|a_{\mu}\right|}-\frac{\left|a_{\mu}\right|}{r^{2}}\right)=N\left(r, \frac{1}{f}\right)+\frac{r}{2} \int_{0}^{r} N\left(x, \frac{1}{f}\right)\left(\frac{1}{x^{2}}-\frac{1}{r^{2}}\right) d x .
$$

using (1.7) (and the analogous formula for poles) in (1.6), we obtain

$$
\begin{aligned}
& \frac{1}{2 \pi} \int_{0}^{2 \pi} \log \left|f\left(r e^{i \theta}\right)\right| \cos \theta d \theta \geqq \cos \gamma\left\{N\left(r, \frac{1}{f}\right)+N(r, f)\right\} \\
& \quad+\frac{r}{2}\left\{\cos \gamma \int_{0}^{r}\left\{N\left(x, \frac{1}{f}\right)+N(x, f)\right\}\left(\frac{1}{x^{2}}-\frac{1}{r^{2}}\right) d x-\left|\frac{f^{\prime}(0)}{f(0)}\right|\right\} .
\end{aligned}
$$

If $r$ is large enough, this implies (1.4) since, by our assumption (1.3), the integral in the right-hand side of (1.8) tends to $+\infty$ as $r \rightarrow+\infty$.

2. Lower bounds for $m(r, f)$.

LEMMA 2. Let $g(z)$ be an absolutely convergent product of primary factors of genus 2.

Assume that the zeros of $g(z)$ lie in the sector $\Delta(\varepsilon)$ defined by

$$
|\arg z| \leqq \frac{\pi}{6}-\varepsilon \quad\left(0<\varepsilon \leqq \frac{\pi}{6}\right) .
$$


Then

$$
\int_{(\pi / 3)-(\varepsilon / 2)}^{(\pi / 3)+(\varepsilon / 2)} \log ^{+}\left|\frac{g\left(r e^{i \theta}\right)}{g\left(-r e^{i \theta}\right)}\right| d \theta \geqq 2 \varepsilon \sin \frac{\varepsilon}{2} r^{3} \int_{0}^{\infty} \frac{n\left(t, \frac{1}{g}\right)}{t^{2}\left(t^{2}+r^{2}\right)} d t .
$$

Proof. Let

$$
E(u, q)=(1-u) \exp \left(u+\frac{u^{2}}{2}+\cdots+\frac{u^{q}}{q}\right),
$$

denote the primary factor of genus $q$; we write $E(u)$ instead of $E(u, 2)$.

It follows from the definitions that

$$
\log \frac{E(u)}{E(-u)}=\operatorname{lon}\left(\frac{1-u}{1+u}\right)+2 u=2 \int_{0}^{u} \frac{t^{2}}{t^{2}-1} d t,
$$

and hence, if

$$
\begin{gathered}
u=r e^{i \phi}, \quad \phi \not \equiv 0(\bmod \pi), \\
\log \left|\frac{E\left(r e^{i \phi}\right)}{E\left(-r e^{i \phi}\right)}\right|=2 \int_{0}^{r} \frac{x^{4} \cos \phi-x^{2} \cos 3 \phi}{x^{4}-2 x^{2} \cos 2 \phi+1} d x .
\end{gathered}
$$

Let $\left\{c_{\nu}\right\}$ be the sequence of the zeros of $g(z)$; putting

$$
\theta_{\nu}=\arg c_{\nu},
$$

we have, by assumption

$$
\left|\theta_{\nu}\right| \leqq \frac{\pi}{6}-\varepsilon
$$

If $z\left(=r e^{i \theta}\right)$ is confined to the sector

$$
\left|\theta-\frac{\pi}{3}\right| \leqq \frac{\varepsilon}{2},
$$

(2.3), (2.4) and (2.5) yield

$$
\begin{aligned}
\log \left|\frac{E\left(\frac{z}{c_{\nu}}\right)}{E\left(\frac{-z}{c_{\nu}}\right)}\right| & =2 \int_{0}^{r /\left|c_{\nu}\right|} \frac{x^{4} \cos \left(\theta-\theta_{\nu}\right)-x^{2} \cos 3\left(\theta-\theta_{\nu}\right)}{x^{4}-2 x^{2} \cos 2\left(\theta-\theta_{\nu}\right)+1} d x \\
& \geqq 2 \sin \frac{\varepsilon}{2} \int_{0}^{r /\left|c_{\nu}\right|} \frac{x^{2}}{1+x^{2}} d x .
\end{aligned}
$$

Hence, in the region defined by (2.5) 


$$
\begin{aligned}
\log ^{+}\left|\frac{g\left(r e^{i \theta}\right)}{g\left(-r e^{i \theta}\right)}\right| & \geqq 2 \sin \frac{\varepsilon}{2} \sum_{\nu=1}^{\infty} \int_{0}^{r /\left|c_{\nu}\right|} \frac{x^{2}}{1+x^{2}} d x \\
& =2 \sin \frac{\varepsilon}{2} r^{3} \int_{0}^{\infty} \frac{n\left(t, \frac{1}{g}\right)}{t^{2}\left(t^{2}+r^{2}\right)} d t
\end{aligned}
$$

and this clearly implies (2.2).

LEMMA 3. Let $f(z)$ be a meromorphic function of genus not greater than 2.

\section{Assume}

(i) that its zeros $\left\{a_{\mu}\right\}$ lie in the region $\Delta(\varepsilon)$ defined by (2.1);

(ii) that its poles $\left\{b_{\nu}\right\}$ lie in the region $\Delta^{*}(\varepsilon)$ defined by

$$
|\arg z-\pi| \leqq \frac{\pi}{6}-\varepsilon \quad\left(0<\varepsilon \leqq \frac{\pi}{6}\right) ;
$$

(iii) $\sum_{\mu} \frac{1}{\left|a_{\mu}\right|}+\sum_{\nu} \frac{1}{\left|b_{\nu}\right|}=+\infty$.

Then

$$
\begin{aligned}
\frac{1}{2 \pi} \int_{(\pi / 3)-(\varepsilon / 2)}^{(\pi / 3)+(\varepsilon / 2)} \log ^{+}\left|\frac{f\left(r e^{i \theta}\right)}{f\left(-r e^{i \theta}\right)}\right| d \theta \\
\geqq \frac{(1-\eta(r))}{2 \pi} \varepsilon \sin \frac{\varepsilon}{2}\left\{N\left(r, \frac{1}{f}\right)+N(r, f)\right\},
\end{aligned}
$$

where $\eta(r) \rightarrow 0$ as $r \rightarrow+\infty$.

Proof. Since the genus of $f(z)$ does not exceed 2, it is possible to represent the function by

$$
f(z)=e^{P(z)} \frac{\Pi E\left(\frac{z}{a_{\mu}}, 2\right)}{\Pi E\left(\frac{z}{b_{\nu}}, 2\right)},
$$

where the polynomial $P(z)$ is of degree not greater than 2 [it is obvious that the infinite products in (2.8) are not necessarily canonical].

Clearly

$$
\frac{f(z)}{f(-z)}=e^{2 P^{\prime}(0) z} \frac{g(z)}{g(-z)},
$$

where 


$$
g(z)=\Pi E\left(\frac{z}{a_{\mu}}, 2\right) \Pi E\left(-\frac{z}{b_{\nu}}, 2\right) .
$$

By (2.9)

$$
\log ^{+}\left|\frac{f\left(r e^{i \theta}\right)}{f\left(-r e^{i \theta}\right)}\right| \geqq \log ^{+}\left|\frac{g\left(r e^{i \theta}\right)}{g\left(-r e^{i \theta}\right)}\right|-2\left|P^{\prime}(0)\right| r,
$$

and the assumptions (i) and (ii) of Lemma 3 enable us to apply Lemma 2 to the function defined by (2.10). We thus obtain

$$
\begin{aligned}
& \int_{(\pi / 3)-(\varepsilon / 2)}^{(\pi / 3)+(\varepsilon / 2)} \log ^{+}\left|\frac{f\left(r e^{i \theta}\right)}{f\left(-r e^{i \theta}\right)}\right| d \theta \\
& \quad \geqq 2 \varepsilon \sin \frac{\varepsilon}{2} r^{3} \int_{0}^{\infty} \frac{n\left(t, \frac{1}{f}\right)+n(t, f)}{t^{2}\left(t^{2}+r^{2}\right)} d t-2 \varepsilon\left|P^{\prime}(0)\right| r .
\end{aligned}
$$

Now

$$
r^{3} \int_{0}^{\infty} \frac{n\left(t, \frac{1}{f}\right)+n(t, f)}{t^{2}\left(t^{2}+r^{2}\right)} d t>\frac{r}{2} \int_{0}^{r} \frac{n\left(t, \frac{1}{f}\right)+n(t, f)}{t^{2}} d t,
$$

and by assumption (iii) the latter integral tends to $+\infty$ as $r \rightarrow+\infty$. Hence (2.11) yields

$$
\begin{aligned}
\frac{1}{2 \pi} \int_{(\pi / 3)-(\varepsilon / 2)}^{(\pi / 3)+(\varepsilon / 2)} \log ^{+} \mid & \frac{f\left(r e^{i \theta}\right)}{f\left(-r e^{i \theta}\right)} \mid d \theta \\
& \geqq(1-\eta(r)) \frac{\varepsilon}{\pi} \sin \frac{\varepsilon}{2} r^{3} \int_{0}^{\infty} \frac{n(t)}{t^{2}\left(t^{2}+r^{2}\right)} d t,
\end{aligned}
$$

where

$$
n(t)=n\left(t, \frac{1}{f}\right)+n(t, f),
$$

and $\eta(r) \rightarrow 0$ as $r \rightarrow+\infty$.

Putting

$$
N(t)=\int_{0}^{t} \frac{n(x)}{x} d x
$$

an integration by parts and obvious estimates yield

$$
\begin{aligned}
\int_{0}^{\infty} \frac{n(t)}{t^{2}\left(t^{2}+r^{2}\right)} d t=\int_{0}^{\infty} N(t) d\left\{-\frac{1}{t\left(t^{2}+r^{2}\right)}\right\} \\
\geqq N(r) \int_{r}^{\infty} d\left\{-\frac{1}{t\left(t^{2}+r^{2}\right)}\right\}=\frac{N(r)}{2 r^{3}} .
\end{aligned}
$$


Using the latter estimate in (2.12), we obtain (2.7).

LEMMA 4. If, in Lemma 3, we restrict the value of the parameter $\varepsilon$ by the inequalities

$$
\frac{9}{10} \frac{\pi}{6} \leqq \varepsilon \leqq \frac{\pi}{6}
$$

then, for all sufficiently large values of $r$,

$$
T(r, f) \geqq(1+A)\left\{N\left(r, \frac{1}{f}\right)+N(r, f)\right\},
$$

where $A(>0)$ is an absolute constant.

The inequality (2.14) still holds if $f(z)$ is replaced by $F(z)$ :

$$
F(z)=e^{S(z)} f(z)
$$

where $S(z)$ is an entire function (which may reduce to a polynomial).

Proof. We apply Lemma 1 to the function $f(z) / f(-z)$ (instead of $f(z))$. By (2.13) and the definition of $\Delta(\varepsilon)$ and $\Delta^{*}(\varepsilon)$, we obtain, for large values of $r$,

$$
\frac{1}{2 \pi} \int_{0}^{2 \pi} \log \left|\frac{f\left(r e^{i \theta}\right)}{f\left(-r e^{i \theta}\right)}\right| \cos \theta d \theta \geqq \cos \left(\frac{\pi}{60}\right)\left\{2 N\left(r, \frac{1}{f}\right)+2 N(r, f)\right\} .
$$

Hence, in view of the trivial relation

$$
\frac{1}{2 \pi} \int_{0}^{2 \pi} \log \left|\frac{f\left(-r e^{i \theta}\right)}{f\left(r e^{i \theta}\right)}\right| d \theta=0
$$

we find, for $r$ large enough,

$$
\begin{gathered}
\frac{1}{2 \pi} \int_{0}^{2 \pi} \log \left|\frac{f\left(-r e^{i \theta}\right)}{f\left(r e^{i \theta}\right)}\right|(1-\cos \theta) d \theta \geqq 2 \cos \frac{\pi}{60}\left\{N\left(r, \frac{1}{f}\right)+N(r, f)\right\}, \\
2 m\left(r, \frac{f(-z)}{f(z)}\right) \geqq \frac{1}{2 \pi} \int_{0}^{2 \pi} \log ^{+}\left|\frac{f\left(r e^{i \theta}\right)}{f\left(-r e^{i \theta}\right)}\right|(1-\cos \theta) d \theta \\
+2 \cos \frac{\pi}{60}\left\{N\left(r, \frac{1}{f}\right)+N(r, f)\right\},
\end{gathered}
$$

$$
\begin{array}{r}
2 m\left(r, \frac{f(-z)}{f(z)}\right) \geqq\left(1-\cos \frac{\pi}{4}\right) \frac{1}{2 \pi} \int_{(\pi / 3)-(\varepsilon / 2)}^{(\pi / 3)+(\varepsilon / 2)} \log ^{+}\left|\frac{f\left(r e^{i \theta}\right)}{f\left(-r e^{i \theta}\right)}\right| d \theta \\
+2 \cos \frac{\pi}{60}\left\{N\left(r, \frac{1}{f}\right)+N(r, f)\right\} .
\end{array}
$$


Using (2.7) and inequalities for the means of Nevanlinna, (2.16) yields

$$
\begin{aligned}
m(r, f(z))+m\left(r, \frac{1}{f(z)}\right) \geqq\{(1-\eta(r)) & \left.\frac{\left(1-\cos \frac{\pi}{4}\right)}{4 \pi} \varepsilon \sin \frac{\varepsilon}{2}+\cos \frac{\pi}{60}\right\} \\
& \times\left\{N\left(r, \frac{1}{f}\right)+N(r, f)\right\},
\end{aligned}
$$

and hence, by Jensen's formula,

$$
\begin{aligned}
2 T(r, f) \geqq\left(1+\cos \frac{\pi}{60}+\right. & \left.\frac{(1-\eta(r))\left(1-\cos \frac{\pi}{4}\right) \varepsilon \sin \frac{\varepsilon}{2}}{4 \pi}\right) \\
& \times\left\{N\left(r, \frac{1}{f}\right)+N(r, f)\right\}(f(0)=1) .
\end{aligned}
$$

Using (2.13), it is easy to obtain an explicit numerical bound for the coefficient of $N(r, 1 / f)+N(r, f)$ in (2.17). Since this bound exceeds 2 , we obtain (2.14).

In order to see that (2.14) holds if $f(z)$ is replaced by $F(z)$, we observe that

$$
m\left(r, e^{S(z)}\right) \leqq T(r, F(z))+T(r, f(z)) \quad(f(0)=1)
$$

Now

$$
T(r, f)=o\left(r^{3}\right) \quad(r \rightarrow+\infty),
$$

because, by assumption, $f(z)$ is of genus not greater than 2 [3; p. 235].

If $S(z)$ is a polynomial of degree not greater than 2, there is nothing to prove since $F(z)$ is still of genus not greater than 2 . In all other cases

$$
X r^{3} \leqq m\left(r, e^{S(z)}\right),
$$

for some $X(>0)$ and $r$ sufficiently large. Hence we obtain the last assertion of the lemma by combining (2.14), (2.18), (2.19), and (2.20).

\section{Proof of Theorem 1.}

Inequality (5) of Theorem 1 follows readily from Lemma 1 and Jensen's theorem: with $\gamma=0$, (1.4) yields

$$
\begin{aligned}
m(r, f)+m\left(r, \frac{1}{f}\right) \geqq N\left(r, \frac{1}{f}\right)+N(r, f), & \\
2 T(r, f) \geqq 2\left\{N\left(r, \frac{1}{f}\right)+N(r, f)\right\} & (f(0)=1),
\end{aligned}
$$

which obviously implies (5). 
The first part of Theorem 1 is contained in the following Lemma 5 which we now state and prove.

Lemma 5. Let $f(z)$ be meromorphic. Assume that there exists an integer $q(\geqq 1)$ such that

$$
\begin{gathered}
\sum_{\mu} \frac{1}{\left|a_{\mu}\right|^{q}}+\sum_{\nu} \frac{1}{\left|b_{\nu}\right|^{q}}=+\infty . \\
\sum_{\mu} \frac{1}{\left|a_{\mu}\right|^{q+1}}+\sum_{\nu} \frac{1}{\left|b_{\nu}\right|^{q+1}}<+\infty .
\end{gathered}
$$

Let $p$ be an odd integer

$$
1 \leqq p \leqq q .
$$

Consider the sectors $\Delta_{k}$ defined by

$$
\left|\arg z-\frac{2 \pi k}{p}\right| \leqq \frac{\pi}{60 q} \quad(k=0,1,2, \cdots, p-1) .
$$

and the sectors $\Delta_{k}^{*}$ defined by

$$
\left|\arg z-\pi-\frac{2 \pi k^{\prime}}{p}\right| \leqq \frac{\pi}{60 q} \quad\left(k^{\prime}=0,1,2, \cdots, p-1\right) .
$$

If every zero of $f(z)$ lies in one of the sectors $\Delta_{k}$ and every pole in one of the sectors $\Delta_{k^{\prime}}^{*}$, then

$$
\limsup _{r \rightarrow \infty} \frac{N\left(r, \frac{1}{f}\right)+N(r, f)}{T(r, f)} \leqq \frac{1}{1+A},
$$

where $A$ is the absolute constant in Lemma 4.

Proof. Consider the odd integer $s$ defined by

$$
s \leqq \frac{q}{p}<s+2
$$

in view of (3.3)

$$
1 \leqq s
$$

Put

$$
l=p s, \quad \omega=\exp \left(\frac{2 \pi i}{l}\right)
$$


Clearly $l$ is a positive odd integer and, by (3.7)

$$
l \leqq q<l+2 p \leqq 3 l .
$$

In view of (3.1) and (3.2), the function $f(z)$ is of the form

$$
f(z)=e^{s(z)} \frac{\Pi E\left(\frac{z}{a_{\mu}}, q\right)}{\Pi E\left(\frac{z}{b_{\nu}}, q\right)},
$$

where $S(z)$ is entire.

Consider now the auxiliary function

$$
G(z)=f(z) f(\omega z) \cdots f\left(\omega^{l-1} z\right)=e^{R\left(z^{l}\right)} \frac{\Pi E\left(\frac{z^{l}}{a_{\mu}^{l}},\left[\frac{q}{l}\right]\right)}{\Pi E\left(\frac{z^{l}}{b_{\nu}^{l}},\left[\frac{q}{l}\right]\right)},
$$

where $R(z)$ is entire and the genus $[q / l]$ of the primary factors is, by (3.9), either 1 or 2.

Putting

$$
\phi_{\mu}=\arg a_{\mu}, \quad \psi_{\nu}=\arg b_{\nu},
$$

our inequalities (3.4), (3.5), and (3.7) imply

$$
\left|\phi_{\mu} l-2 \pi k s\right| \leqq \frac{\pi}{60}, \quad\left|\psi_{\nu} l-\pi l-2 \pi k^{\prime} s\right| \leqq \frac{\pi}{60}
$$

We also notice that our assumptions prevent the possibility of cancellation between the zeros of one of the functions $f\left(\omega^{j} z\right)(j=0,1, \cdots$, $l-1)$ and the poles of another of these functions. Hence

$$
N(r, G(z))=l N(r, f), \quad N\left(r, \frac{1}{G(z)}\right)=l N\left(r, \frac{1}{f}\right) .
$$

Put

$$
H(u)=e^{R(u)} \frac{\Pi E\left(\frac{u}{a_{\mu}^{l}},\left[\frac{q}{l}\right]\right)}{\Pi E\left(\frac{u}{b_{\nu}^{l}},\left[\frac{q}{l}\right]\right)},
$$

and rewrite (3.10) as

$$
G(z)=H\left(z^{l}\right) \text {. }
$$

The inequalities (3.11), the assumption (3.1), and the first of the 
inequalities (3.9) show that it is possible to apply Lemma 4 to $H(u)$ (instead of $f(z)$ ). Hence

$$
T(r, H(u)) \geqq(1+A)\left\{N\left(r, \frac{1}{H(u)}\right)+N(r, H(u))\right\} \quad\left(r \geqq r_{0}\right) .
$$

On the other hand, the fundamental definitions of Nevanlinna's theory show that, for any meromorphic function $w(z)$ :

$$
N\left(r, w\left(z^{l}\right)\right)=N\left(r^{l}, w(z)\right), \quad T\left(r, w\left(z^{l}\right)\right)=T\left(r^{l}, w(z)\right),
$$

so that (3.13) and (3.14) yield

$$
T(r, G(z)) \geqq(1+A)\left\{N\left(r, \frac{1}{G(z)}\right)+N(r, G(z))\right\} \quad\left(r^{2} \geqq r_{0}\right) .
$$

Since

$$
l T(r, f) \geqq T(r, G(z)),
$$

we see that (3.6) follows from (3.12) and (3.15).

We obtain the first part of Theorem 1 by taking $p=1$ in Lemma 5 .

4. Proof of the Corollaries. Corollary 1.1 follows trivially from the inequalities (4) and (5) and the definition of deficiency.

Corollary 1.2 is contained in the following.

Lemma 6. Let $f(z)$ be entire. Modify the assumptions of Lemma 5 by:

(i) omitting all reference to poles;

(ii) omitting the restriction that $p$ be odd ( $p$ may be any integer satisfying the inequality (3.3)).

Then (3.6) still holds.

The proof of Lemma 5 also yields Lemma 6 provided the integer $s$ (even or odd) is defined by

$$
s \leqq \frac{q}{p}<s+1
$$

instead of (3.7). The definitions (3.8) remain unchanged and (3.9) takes the sharper form

$$
l \leqq q<2 l .
$$

The other changes in the proof are obvious and need not be mentioned here.

We obtain Corollary 1.2 by taking $p=2$, in Lemma 6 . 
5. Best possible character of the conditions (2) and (6). Let

$$
s_{1}, s_{2}, s_{3}, \cdots
$$

be a sequence of integers such that

$$
s_{1} \geqq 2, \quad s_{\lambda+1}>2 s_{\lambda}
$$

$$
(\lambda=1,2,3, \cdots)
$$

Consider the entire function

$$
f(z)=\prod_{\lambda=1}^{\infty} \prod_{m=s_{\lambda}}^{2 s_{\lambda}}\left(1-\frac{z}{m(\log m)^{2}}\right) .
$$

Denoting by $\left\{a_{\mu}\right\}$ the sequence of the zeros of $f(z)$, elementary estimates yield

$$
\sum_{\mu} \frac{1}{a_{\mu}}<+\infty, \quad \sum_{\mu} \frac{1}{a_{\mu}^{\kappa}}=+\infty
$$

These relations hold for every choice of the sequence (5.1). Hence we may take the ratios $s_{\lambda+1} / s_{\lambda}$ to be rapidly increasing with $\lambda$ and, using the well-known formula $[4 ;$ p. 271]:

$$
\log M(r, f)=r \int_{0}^{\infty} \frac{n\left(t, \frac{1}{f}\right)}{t(t+r)} d t,
$$

choose (5.1) so that

$$
\liminf _{r \rightarrow \infty} \frac{\log T(r, f)}{\log r} \leqq \liminf _{r \rightarrow \infty} \frac{\log \log M(r)}{\log r}=0 .
$$

It is sufficient to choose the sequence (5.1) in such a way that, for some arbitrarily large $u, n(t, 1 / f)$ is constant in $u \leqq t \leqq e^{u}$.

Hence, putting

$$
F(z)=\frac{f(z)}{f(-z)},
$$

$$
\liminf _{r \rightarrow \infty} \frac{\log T(r, F(z))}{\log r}=0 .
$$

It has been shown elsewhere $[1 ; p .297$, Theorem 4] that the condition (5.5) implies

$$
\delta(\tau, F(z))=0,
$$

except possibly for a single value of $\tau$, finite or infinite. 
Hence the inequalities

$$
\delta(0, F(z))>0, \quad \delta(\infty, F(z))>0,
$$

are both impossible since one of them would imply the other one. We thus have

$$
1=\limsup _{r \rightarrow \infty} \frac{N(r, F)}{T(r, F)}=\frac{1}{2} \limsup _{r \rightarrow \infty} \frac{N\left(r, \frac{1}{F}\right)+N(r, F)}{T(r, F)},
$$

although $F(z)$ satisfies all the conditions of Theorem 1 except (2) which is replaced by the weaker condition (5.3).

Similarly, (5.4) and Theorem 4 of [1] yield

$$
\delta(\tau, f(z))=0
$$

and hence, putting

$$
F^{*}(z)=f\left(z^{2}\right)
$$

we have

$$
\delta\left(\tau, F^{*}(z)\right)=0
$$$$
(\tau \neq \infty)
$$

In particular $\delta\left(0, F^{*}(z)\right)=0$, although $F^{*}(z)$ satisfies all the conditions of Corollary 1.2 except (6) which is replaced by a weaker condition analogous to (5.3).

6. Proof of Theorem 2. Our proof is a straightforward consequence of Lemma 6 and of a classical theorem of H. Weyl [5; p. 335, Satz 16].

We consider the arguments $\omega_{j}$ of the radii carrying the zeros of $f(z)$ and assume

$$
\omega_{0}=0
$$

this is clearly no restriction.

Let $k+1(0 \leqq k \leqq m)$ be the maximum number of linearly independent elements among

$$
2 \pi, \omega_{1}, \omega_{2}, \cdots \omega_{m} .
$$

Renumbering, if necessary, the $\omega$ 's we may assume:

(i) that a relation such as

$$
\mu_{0} 2 \pi+\sum_{j=1}^{k} \mu_{j} \omega_{j}=0,
$$

is impossible for integral values of the $\mu^{\prime}$ s, not all zero;

(ii) if $k<m$, there exist integers $n_{l_{j}}$ and $\sigma(>0)$ such that 


$$
\sigma \omega_{l}=2 \pi n_{l 0}+\sum_{j=1}^{k} n_{l_{j}} \omega_{j} \quad(l=k+1, \cdots, m) .
$$

Put

$$
M_{l}=\sum_{j=1}^{k}\left|n_{l_{j}}\right|
$$

and

$$
M=\sup \left\{\sigma, M_{k+1}, M_{k+2}, \cdots, M_{m}\right\} .
$$

Since no relation such as (6.2) is possible, Weyl's theorem asserts the existence of a sequence

$$
\lambda_{1}, \lambda_{2}, \lambda_{3}, \cdots
$$

of increasing integers such that

$$
\left|\lambda_{s} \omega_{j}-L_{s j} 2 \pi\right| \leqq \frac{\pi}{120 M} \quad(j=1,2, \cdots k ; s=1,2,3, \cdots),
$$

where the $L_{s,}$ are integers. Weyl's theorem also asserts that the sequence (6.5) has a positive density. The latter property is unnecessarily precise for our purposes; we only need the obvious implication

$$
\lambda_{s+1}<2 \lambda_{s}
$$$$
\left(s \geqq s_{0}\right) .
$$

We set

$$
K=\sigma \lambda_{s_{0}}
$$

and observe that the integer $K$ depends only on the $\omega$ 's.

By the assumptions (9) and (10), there exists an integer $q$ such that

$$
q \geqq K, \quad \sum_{\mu} \frac{1}{\left|a_{\mu}\right|^{q}}=+\infty, \quad \sum_{\mu} \frac{1}{\left|a_{\mu}\right|^{q+1}}<+\infty .
$$

Define $h$ by the inequalities

$$
\sigma \lambda_{h} \leqq q<\sigma \lambda_{h+1}
$$

In view of the definition of $K$ and (6.7)

$$
q<2 \sigma \lambda_{h} \text {. }
$$

We now obtain Theorem 2 by verifying that Lemma 6 may be applied with the value of $q$ chosen above and

$$
p=\sigma \lambda_{h} .
$$

It is clear that we only have to ascertain that the zeros of $f(z)$ lie in regions such as (3.4) with $p$ defined by (6.10). 
Using (6.6) and (6.4) in (6.3), we obtain

$$
\left|\sigma \lambda_{h} \omega_{l}-\Lambda_{h l} 2 \pi\right| \leqq \frac{\pi}{120} \quad(l=k+1, k+2, \cdots m),
$$

where the $\Lambda$ 's are integers.

By (6.6) and (6.4), it is clear that (6.11) holds also for $l=1,2, \cdots k$, with

$$
\Lambda_{h l}=\sigma L_{h l} \quad(l=1,2, \cdots k) .
$$

Hence, by (6.9), (6.10) and (6.11)

$$
\left|\omega_{l}-\frac{\Lambda_{h l} 2 \pi}{p}\right| \leqq \frac{\pi}{60 q} \quad(l=1,2, \cdots m) .
$$

This shows that the location of zeros allows the application of Lemma 6. Theorem 2 is an immediate consequence.

\section{REFERENCES}

1. A. Edrei and W. H. J. Fuchs, On the growth of meromorphic functions with several deficient values, Trans. Amer. Math. Soc. 93 (1959), 292-328.

2. — On the maximum number of deficient values of certain classes of functions, Air Force Technical Note, AFOSR TN 60-402 (April 1960).

3. R. Nevanlinna, Eindeutige Analytische Funktionen, 2nd ed., Berlin, 1953.

4. E. C. Titchmarsh, The theory of functions 2d ed., Oxford, 1950.

5. H. Weyl, Über die Gleichverteilung von Zahlen mod. Eins, Math. Annalen 77 (1916), 313-352.

SYRACUSE UNIVERSITY AND CORNELL UNIVERSITY 



\section{PACIFIC JOURNAL OF MATHEMATICS}

\section{EDITORS}

Ralph S. Phillips

Stanford University

Stanford, California

F. H. BrownelL

University of Washington

Seattle 5 , Washington
A. L. Whiteman

University of Southern California Los Angeles 7. California

L. J. PAIGE

University of California

Los Angeles 24, California

ASSOCIATE EDITORS
E. F. BECKENBACH
D. DERRY
H. L. ROYDEN
E. G. STRAUS
T. M. CHERRY
M. OHTSUKA
E. SPANIER
F. WOLF

\section{SUPPORTING INSTITUTIONS}

UNIVERSITY OF BRITISH COLUMBIA

CALIFORNIA INSTITUTE OF TECHNOLOGY

UNIVERSITY OF CALIFORNIA

MONTANA STATE UNIVERSITY

UNIVERSITY OF NEVADA

NEW MEXICO STATE UNIVERSITY

OREGON STATE COLLEGE

UNIVERSITY OF OREGON

OSAKA UNIVERSITY

UNIVERSITY OF SOUTHERN CALIFORNIA
STANFORD UNIVERSITY

UNIVERSITY OF TOKYO

UNIVERSITY OF UTAH

WASHINGTON STATE COLLEGE,

UNIVERSITY OF WASHINGTON

AMERICAN MATHEMATICAL SOCIETY

CALIFORNIA RESEARCH CORPORATION

HUGHES AIRCRAFT COMPANY

SPACE TECHNOLOGY LABORATORIES

NAVAL ORDNANCE TEST STATION

Printed in Japan by International Academic Printing Co., Ltd., Tokyo, Japan

Reprinted 1966 in the United States of America 


\section{Pacific Journal of Mathematics}

\section{Vol. 11, No. $1 \quad$ November, 1961}

A. A. Albert, Generalized twisted fields ............................ 1

Richard Arens, Operational calculus of linear relations ................... 9

John Herbert Barrett, Disconjugacy of a self-adjoint differential equation of the fourth order ....................................... 25

Paul Richard Beesack, Hardy's inequality and its extensions ............... 39

Julius Rubin Blum and David Lee Hanson, On invariant probability measures.

II .............................................

Robert Allen Bonic, Symmetry in group algebras of discrete groups.......... 73

R. Creighton Buck, Multiplication operators ...................... 95

Jack Gary Ceder, Some generalizations of metric spaces ................. 105

Meyer Dwass, Random crossings of cumulative distribution functions ......... 127

Albert Edrei, Wolfgang H. J. Fuchs and Simon Hellerstein, Radial distribution and

deficiencies of the values of a meromorphic function ............... 135

William Cassidy Fox, Harmonic functions with arbitrary local singularities ..... 153

Theodore Thomas Frankel, Manifolds with positive curvature ............... 165

Avner Friedman, A strong maximum principle for weakly subparabolic

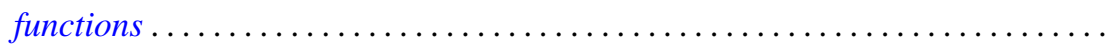

Watson Bryan Fulks and J. O. Sather, Asymptotics. II. Laplace's method for

multiple integrals ......................................

Adriano Mario Garsia and Eugene Richard Rodemich, An embedding of Riemann

surfaces of genus one ..................................... 193

Irving Leonard Glicksberg, Weak compactness and separate continuity......... 205

Branko Grünbaum, On a conjecture of H. Hadwiger .................. 215

Frank J. Hahn, On the action of a locally compact group on $E_{n} \ldots \ldots \ldots \ldots \ldots . . \ldots 221$

Magnus R. Hestenes, Relative hermitian matrices ..................... 225

G. K. Kalisch, On similarity invariants of certain operators in $L_{p} \ldots \ldots \ldots \ldots .247$

Yitzhak Katznelson and Walter Rudin, The Stone-Weierstrass property in Banach

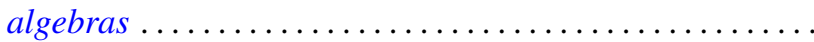

Samir A. Khabbaz, The subgroups of a divisible group $G$ which can be represented as intersections of divisible subgroups of $G \ldots \ldots \ldots \ldots \ldots \ldots \ldots \ldots \ldots . \ldots \ldots 7$

Marvin Isadore Knopp, Construction of a class of modular functions and forms .......................................... 275

Charles Alan McCarthy, Commuting Boolean algebras of projections .......... 295

T. M. MacRobert, Transformations of series of E-functions ................ 309

Heinz Renggli, An inequality for logarithmic capacities ................. 313

M. S. Robertson, Applications of the subordination principle to univalent functions .......................................... 315

David Sachs, Partition and modulated lattices ..................... 325

Frank S. Scalora, Abstract martingale convergence theorems ............... 347

Elbert A. Walker, Torsion endomorphic images of mixed Abelian groups ........ 375

Morgan Ward, The prime divisors of Fibonacci numbers................. 379

Charles R. B. Wright, On the nilpotency class of a group of exponent four....... 387 